\title{
Inhibitory effects of ubiquitination of synoviolin by PADI4
}

\author{
SATOKO ARATANI ${ }^{1-3}$, HIDETOSHI FUJTAA $^{1,2}$, NAOKO YAGISHITA ${ }^{4}$, YOSHIHISA YAMANO ${ }^{4}$, \\ YUKARI OKUBO ${ }^{3,5}$, KUSUKI NISHIOKA ${ }^{6}$ and TOSHIHIRO NAKAJIMA ${ }^{1-3,7,8}$ \\ ${ }^{1}$ Department of Locomotor Science, Institute of Medical Science, Tokyo Medical University; \\ ${ }^{2}$ Department of Future Medical Science, Tokyo Medical University, Tokyo 160-8402; ${ }^{3}$ Physician, \\ Student and Researcher Support Center, Tokyo Medical University, Tokyo 160-0023; ${ }^{4}$ Department of \\ Rare Diseases Research, Institute of Medical Science, St. Marianna University School of Medicine, Kawasaki, \\ Kanagawa 216-8512; ${ }^{5}$ Department of Dermatology, Tokyo Medical University, Tokyo 160-0023; ${ }^{6}$ Institute of \\ Medical Science, Tokyo Medical University, Tokyo 160-8402; ${ }^{7}$ Integrated Gene Editing Section (iGES), \\ Tokyo Medical University Hospital, 160-0023 Tokyo; ${ }^{8}$ Bayside Misato Medical Center, Kochi, Kochi, 780-0832, Japan
}

Received February 11, 2016; Accepted February 9, 2017

DOI: $10.3892 / \mathrm{mmr} .2017 .7764$

\begin{abstract}
Rheumatoid arthritis (RA) is a chronic inflammatory articular disease that is characterized by synovial hyperplasia. A number of signaling pathways are associated with the development and induced symptoms of RA. Notably, patients with RA have increased protein citrullination and generation of auto-antibodies against citrullinated proteins. Genome wide association studies have revealed that peptidyl-arginine deiminase 4 (PADI4) is an enzyme implicated in citrullination in the RA synovium. Autoantibodies targeting citrullinated proteins are used as diagnostic markers in patients with RA. The functions associated with citrullinated proteins are thought to induce autoimmunity, however, the regulatory mechanisms of citrullination via PADI4 are unclear. The group has previously cloned an E3 ubiquitin ligase, synoviolin (SYVN1), from the RA synovium, demonstrating that SYVN1 serves critical roles in synovial hyperplasia. The data indicated that the endoplasmic reticulum (ER) associated degradation system, which involves SYVN1, may have important roles in the proliferation of synoviocytes. In addition, ubiquitination by SYVN1 is associated with fibrosis, inflammation and cytokine production via the regulation of ER stress signals and quality control of proteins. The present study investigated the crosstalk between the representative post-translational signaling processes, citrullination and ubiquitination. The results revealed that PADI4 interacted with SYVN1 directly and that overexpression of PADI4 suppressed the ubiquitination of proteins. Thus, a reduction in ER stress induced by PADI4 may abrogate
\end{abstract}

Correspondence to: Professor Toshihiro Nakajima, Department of Locomotor Science, Institute of Medical Science, Tokyo Medical University, 6-1-1 Shinjuku, Shinjuku-ku, Tokyo 160-8402, Japan E-mail: marlin@tokyo-med.ac.jp

Key words: rheumatoid arthritis, post-transcriptional modification, ubiquitination, citrullination, synoviolin, peptidyl-arginine deiminase 4 the initiation of chronic RA by suppressing the proliferative signals of RA synoviocytes.

\section{Introduction}

Rheumatoid arthritis (RA) is one of the most common articular diseases and is characterized by synovial hyperplasia, which impairs quality of life. The estimated prevalence of RA is 0.5 to $1 \%$ worldwide $(1,2)$. The pathological features of RA include overgrowth of synoviocytes, chronic inflammation, destruction of cartilage and bone and terminal-phase tissue fibrosis $(3,4)$. Biological agents, such as inhibitors of tumor necrosis factor and interleukin-6, have recently been developed to be used in RA treatments and have exhibited beneficial therapeutic effects (5). However, these therapies are expensive and may not be efficacious in a significant number of RA patients.

Posttranslational modifications are crucial in the regulation of protein functions by altering protein structure and interactions (6). In RA, recent studies have focused on the impact of citrullination (7-9). Citrullination results in the loss of a positive charge and altered biochemical features $(10,11)$. Citrulline residues are synthesized by the deamination of arginine residues using the $\mathrm{Ca}^{2+}$-dependent enzyme, peptiydylarginine deiminase (PADI). The PADI family consists of five members, PADI 1 to 4 and 6 , which exhibit tissue specific expression (12). Patients with RA have increased levels of citrullinated proteins, including vimentin, fibrinogen, collagen and auto-antibodies against citrullinated protein antibodies (ACPAs) are thought to be synthesized (13). Serum from the majority of patients with RA contains auto-antibodies against a number of proteins and ACPAs. ACPA is reported to have high sensitivity (60\%) and specificity (90\%) as a clinical diagnostic biomarker for RA (14-16).

PADI2 and PADI4 are expressed in the synovial tissue of patients with RA and other types of arthritis (17-19). PADI4 mediated citrullination has been implicated in a number of inflammatory autoimmune diseases, including RA, lupus, colitis and multiple sclerosis $(20,21)$. In addition, PADI4 is a RA-susceptibility gene, which was identified in genome-wide 
association studies in patients with RA. The PADI4 haplotype is associated with RA, and stabilization of PADI4 mRNA leads to increased expression $(22,23)$. PADI4 is a unique enzyme in the PADI family due to its subcellular localization; other PADI family members usually localize in the cytoplasm, whereas PADI4 is also present in the nucleus (24). In addition, PADI4 competes with arginine $\mathrm{N}$-methyltransferase and catalyzes citrullination of arginine residues in histones. Furthermore, PADI4 promotes decondensation of chromatin structure $(25,26)$ and activates the transcription of a number of genes and neutrophil extracellular traps $(27,28)$. Citrullination by PADI4 has been demonstrated to regulate protein localization (29).

In addition to citrullination, protein ubiquitination is important in the onset, pathogenesis and the associated symptoms of RA. Endoplasmic reticulum (ER) stress signals are associated with inflammation (30). ER stress signals, which are triggered by an accumulation of unfolded or misfolded proteins in the ER, induce a protective response known as the unfolded protein response in order to maintain cellular homeostasis $(31,32)$. Accumulated misfolded proteins are degraded through the ubiquitin-proteasome system in the cytoplasm. Secreted and membrane proteins are synthesized in the ER, and pro-inflammatory cytokines and extracellular matrix proteins have been revealed to be associated with ER stress in patients with RA (33-35).

The E3 ubiquitin ligase, synoviolin (SYVN1), has been identified in the synovial tissue of patients with RA. A previous study demonstrated that the expression levels of SYVN1 increased in the RA synovium when compared with that in osteoarthritis (36). SYVN1, a mammalian homologue of Hrd1p/Der3p, serves important roles in the ER-associated protein degradation (ERAD) pathway. SYVN1 deficient mice exhibited resistance to collagen-induced arthritis, by contrast, overexpression of SYVN1 induced arthropathy (36). In addition, SYVN1 has been revealed to control the tumor suppressor p53 as a substrate and negatively regulate its biological functions in transcription, the cell cycle and apoptosis $(37,38)$. As the expression of SYVN1 is higher in patients that do not respond to treatment with infriximab, SYVN1 may have applications as a biomarker to facilitate the selection of suitable treatments $(39,40)$. Thus, these previous studies indicated that SYVN1 may have crucial roles in synovial cell hyperplasia in patients with RA. Therefore, the overexpression of SYVN1 in patients with RA may result in a hyper-ERAD state, and in turn, RA may be a disease of the ERAD system (41).

SYVN1 is implicated in a number of diseases in addition to RA. It has been demonstrated that SYVN1 is associated with liver and lung fibrosis (42-44) and obesity in mouse models (45). Analysis of post-neonatal SYVN1-knockout mice has indicated that SYVN1 is associated with energy metabolism and mitochondrial biosynthesis through peroxisome proliferator-activated receptor gamma coactivator $1 \beta$ (PGC-1 $\beta$ ) degradation (45). Previous studies have identified a number of small molecules that inhibit SYVN1 auto-ubiquitination activity, suppress synoviocyte proliferation in patients with RA and reduce the occurrence of arthritis in an RA model mouse (46). In addition, these inhibitors have been demonstrated to block the expression of fibrosis-associated factors $(42,44)$ and reduce body weight and white adipose tissue (45). Thus, SYVN1 may represent a potential therapeutic target.
Citrullinated proteins exhibit altered conformations due to the increased hydrophobicity of citrulline residues, which may be recognized as autoimmune antigens (11). Thus, it may be hypothesized that accumulation of citrullinated proteins is associated with the ubiquitin-proteasome system, a pathway that is induced by unfolded proteins. Therefore, the present study investigated the interactions between ubiquitination and citrullination.

\section{Materials and methods}

Plasmids. The sequence of full-length human PADI4 was amplified from the cDNA of HL-60 cells obtained from American Type Culture Collection (ATCC; cat. no. CCL240; Manassas, VA, USA) using the following primers: Forward 5'-CGCGAATTCATGGCCCAGGGGACATTGAT-3' and reverse 5'-GCGGTCGACTCAGGGCACCATGTTCCACC-3'. Mutant PADI4 (p.C645S), which is characterized by a Cys to Ser substitution at amino acid (aa) 645 in the calcium binding site (26), was amplified using the following primers: forward 5'-GAGGTGCACTCCGGCACCAACGT-3' and reverse 5'-ACGTTGGTGCCGGAGTGCACCTC-3', from the wild-type PADI4. The target fragments were amplified using TaKaRa Ex Taq ${ }^{\circledR}$ DNA Polymerase (Takara Biotechnology Co., Ltd., Dalian, China) under the following conditions: 30 cycles at $94^{\circ} \mathrm{C}$ for $30 \mathrm{sec}$, at $55^{\circ} \mathrm{C}$ for $30 \mathrm{sec}$ and at $72^{\circ} \mathrm{C}$ for 1 min, using the GeneAmp PCR System 9700 (Thermo Fisher Scientific, Inc., Waltham, MA, USA), and were then cloned into the FLAG-tagged pcDNA3 vector (Invitrogen; Thermo Fisher Scientific, Inc.).

Immunoprecipitation assay. Immunoprecipitation assays were performed on HEK 293 cells, as previously described (45). Briefly, HEK293 cells $\left(4 \times 10^{6}\right)$, obtained from ATCC (cat. no. CRL-1573), were transfected with FLAG (control cells) or SYVN1-FLAG constructs with HA-PADI4 (experimental cells). Cells were lysed in lysis buffer, containing $50 \mathrm{mM}$ Tris- $\mathrm{HCl}(\mathrm{pH} 8.0), 150 \mathrm{mM} \mathrm{NaCl}, 1 \mathrm{mM}$ EDTA, 1\% NP-40, 1 mM dithiothreitol (DTT), $10 \mu \mathrm{M}$ MG-132, and protease inhibitors, for $30 \mathrm{~min}$ at $4^{\circ} \mathrm{C}$. Whole cell extracts (WCE) were incubated with $2 \mu \mathrm{g}$ of anti-FLAG (M2) antibodies in $1 \mathrm{ml}$ of buffer A (20 mM Tris- $\mathrm{HCl}$, $\mathrm{pH} 7.5,100 \mathrm{mM} \mathrm{NaCl}, 1 \mathrm{mM}$ EDTA, $1 \mathrm{mM}$ DTT, $0.1 \%$ NP40, 5\% glycerol, $10 \mu \mathrm{M} \mathrm{MG}-132,1 \mu \mathrm{g} / \mathrm{ml}$ aprotinin and $1 \mu \mathrm{g} / \mathrm{ml}$ leupeptin) for $4 \mathrm{~h}$ at $4^{\circ} \mathrm{C}$. Proteins precipitated with anti-FLAG antibody were subjected to $7.5 \%$ SDS-PAGE and detected using western blot analysis with anti-HA or anti-FLAG antibodies.

In vivo ubiquitination assay. HEK293 cells $\left(4 \times 10^{6}\right)$ were transfected with HA-ubiquitin, FLAG-PADI4 and SYVN1-FLAG expression plasmids. Following incubation for $24 \mathrm{~h}$, cells were treated with $10 \mu \mathrm{M}$ MG-132 for $2 \mathrm{~h}$. Cell extracts were prepared as described in the immunoprecipitation assay methodology and separated by $7.5 \%$ SDS-PAGE. Ubiquitinated proteins in WCE were detected using western blot analysis with anti-HA, anti-SYVN1 and anti-PADI4 antibodies, as described in the western blot analysis sub-section. Anti-SYVN1 antibodies detect endogenous proteins, however, anti-PADI4 antibodies do not. 
For auto-ubiquitination of SYVN1 in vivo, HEK293 cells were transfected with HA-ubiquitin, FLAG-PADI4 and His-SYVN1. Following treatment with $10 \mu \mathrm{M}$ MG-132 for $2 \mathrm{~h}$, cells $\left(4 \times 10^{6}\right)$ were harvested and lysed in the lysis buffer. His-tagged SYVN1 was purified by incubation with Ni-Sepharose chromatography resin (GE Healthcare Life Sciences) for $5 \mathrm{~h}$ at $4^{\circ} \mathrm{C}$ and elution using buffer B $(20 \mathrm{mM}$ Tris- $\mathrm{HCl}$ pH 7.5, 0.5 M NaCl, 1 mM EDTA, $1 \%$ Triton X-100, $1 \mathrm{mM}$ DTT, $10 \mu \mathrm{M}$ MG-132 and protease inhibitors). Proteins were subjected to $7.5 \%$ SDS-PAGE and detected using western blot analysis with anti-HA, anti-His and anti-FLAG antibodies, as described in the western blot analysis sub-section.

Pull-down assay.Pull-down assay was performed as previously described (45). Glutathione S-transferase (GST)-fused SYVN1 deletion mutant proteins were expressed in Escherichia coli (E. coli) and purified using glutathione sepharose beads. HA-tagged PADI4 was expressed using the T7 in vitro transcription/translation system (Promega Corporation, Madison, WI, USA) for $1 \mathrm{~h}$ at $30^{\circ} \mathrm{C}$. GST-SYVN1 was incubated with HA-PADI4 in $1 \mathrm{ml}$ buffer A (20 mM Tris- $\mathrm{HCl}, \mathrm{pH}$ 8.0, $100 \mathrm{mM} \mathrm{NaCl}, 1 \mathrm{mM}$ EDTA, $1 \mathrm{mM}$ DTT, 0.1\% NP40, 5\% glycerol, $10 \mu \mathrm{M}$ MG-132, $1 \mu \mathrm{g} / \mathrm{ml}$ aprotinin, and $1 \mu \mathrm{g} / \mathrm{ml}$ leupeptin) for $6 \mathrm{~h}$ at $4^{\circ} \mathrm{C}$. Following washing with buffer $\mathrm{A}$, bound proteins were fractionated by $10 \%$ SDS-PAGE and detected using western blot analysis with anti-HA antibodies, as described in the western blot analysis sub-section.

Western blot analysis. Proteins were separated by SDS-PAGE and transferred onto polyvinylidene difluoride membranes. The membranes were blocked with $5 \%$ milk in TBS buffer with $0.1 \%$ Tween-20 (TBS-T) at room temperature for $1 \mathrm{~h}$, and incubated with the primary antibodies at room temperature for $1 \mathrm{~h}$. The following primary antibodies were used: Anti-FLAG (M2; cat. no. F3165; 1:5,000), anti-human influenza hemagglutinin (HA)-tag (3F10; cat. no. 11867423001; 1:5,000), obtained from Sigma-Aldrich; Merck KGaA (Darmstadt, Germany); anti-polyhistidine (His; cat. no. H-15; 1:1,000; Santa Cruz Biotechnology, Inc., Dallas, TX, USA) and anti-SYVN1 rabbit polyclonal antibodies $(1: 5,000)$, established in our laboratory as previously described $(37,45)$. Anti-PADI4 polyclonal antibodies $(1: 1,000)$ were generated by injection of antigen peptides coding PADI4 (aa 128-140) to a rabbit (Protein Purify, Gunma, Japan). Following washing with TBS-T, the membranes were incubated with horseradish peroxidase-conjugated anti-mouse (1:50,000; cat. no. A5278; Sigma-Aldrich; Merck KGaA), anti-rabbit (1:75,000; cat. no. A9169; Sigma-Aldrich; Merck $\mathrm{KGaA})$ or anti-rat $\operatorname{IgG}$ secondary antibody (1:75,000; cat. no. 112-035-062; Jackson ImmunoResearch Laboratories, Inc., West Grove, PA, USA) at room temperature for $40 \mathrm{~min}$. The protein bands were visualized using the enhanced chemiluminescence Amersham ECL Select Western Blotting Detection system (GE Healthcare Life Sciences, Chalfont, UK).

\section{Results}

Interactions between SYVN1 and PADI4. To assess the association between PADI4 and ubiquitination, immunoprecipitation assays were performed to determine whether PADI4 interacted with SYVN1, which represents the E3 ligase in RA.

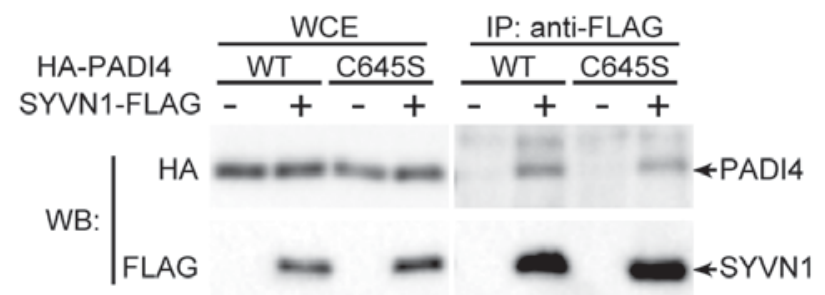

Figure 1. Interactions between SYVN1 and PADI4. Immunoprecipitation of SYVN1 and PADI4. Lysates of HEK293 cells expressing FLAG-tagged SYVN1 and HA-tagged PADI4 were immunoprecipitated using anti-FLAG antibodies. Proteins were detected with anti-HA antibodies. SYVN, synoviolin; PADI4, peptidyl-arginine deiminases 4; HA, human influenza hemagglutinin; WCE, whole cell extract; WB, western blot analysis.

A

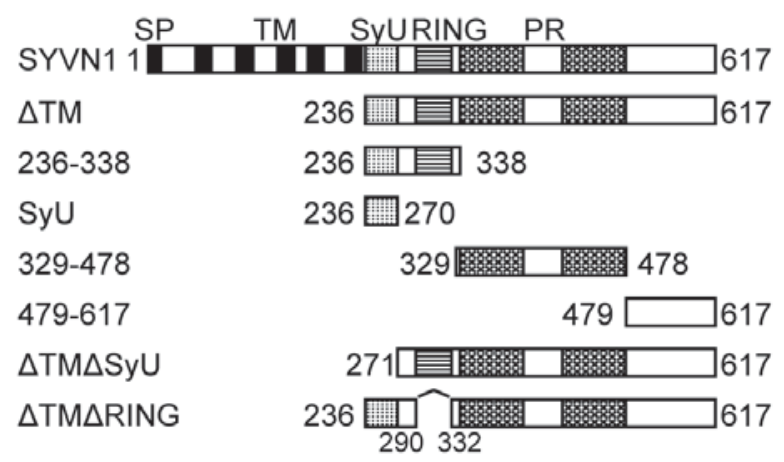

B

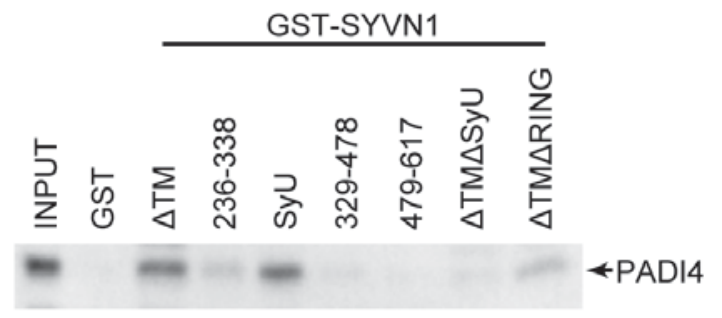

Figure 2. Identification of the PADI4 binding domain of SYVN1. (A) Schematic representation of SYVN1 deletion mutants. (B) Pull-down assay with GST-SYVN1 deletion mutants and HA-PADI4. PADI4, peptidyl-arginine deiminases 4; SYVN, synoviolin; GST, glutathione S-transferase; HA, human influenza hemagglutinin; TM, transmembrane; SyU, SYVN1 unique domain; RING, Really Interesting New Gene finger domain; PR, proline rich domain.

HEK293 cells were transfected with FLAG-tagged SYVN1 and HA-tagged PADI4 expression vectors. The complexes were precipitated with anti-FLAG antibodies for SYVN1 and were detected using anti-HA antibodies. FLAG-tagged SYVN1 was revealed to interact with PADI4 (Fig. 1).

The PADI4 binding region in the SYVN1 protein was then identified to further elucidate the association with PADI4. SYVN1 contains 4 domains, the Really Interesting New Gene (RING) finger domain, the SYVN1 unique domain (SyU) and two proline riche domains. The SyU domain is responsible for interactions with p53 and PGC-1 $\beta(37,45)$. A series of deletion mutants of SYVN1 (Fig. 2A) were expressed as GST-fused proteins in E. coli. GST-fused SYVN1 deletion mutants were incubated with HA-tagged PADI4, which was expressed using the in vitro translation system. As a result, GST-fused SYVN1 lacking the transmembrane domain (SYVN1 $\Delta T M$ ) 


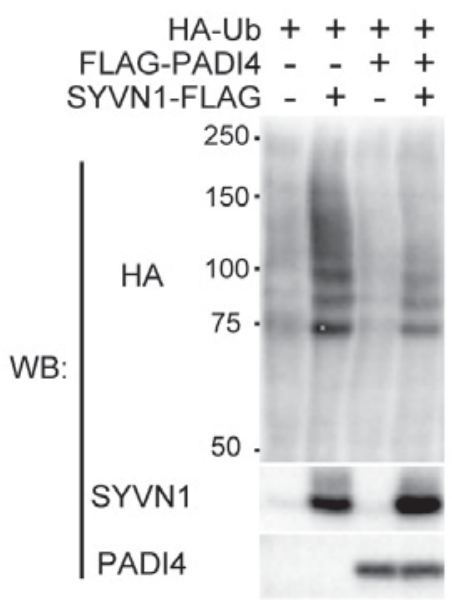

Figure 3. Inhibition of ubiquitination by PADI4. HEK293 cells were transfected with HA-ubiquitin, FLAG-PADI4 and SYVN1-FLAG. Whole-cell extracts were analyzed by western blotting with anti-HA (top blot), anti-SYVN1 (middle blot) and anti-PADI4 (bottom blot) antibodies. The bands detected with anti-SYVN1 in lanes 2 and 4 were endogenous SYVN1 Ub, ubiquitin; PADI4, peptidyl-arginine deiminases 4; SYVN, synoviolin; HA, human influenza hemagglutinin; WB, western blot analysis.

could bind PADI4. The region from aa 236 to 338, which contains the RING finger domain, and the SyU domain could bind PADI4. The region from aa 339 to 478 , which contains proline rich domains, may interact with PADI4 weakly. In addition, the mutants lacking the RING finger domain (SYVN1 $\Delta \mathrm{TM} \Delta \mathrm{RING}$ ) and the SyU domain could bind with PADI4. In contrast, the C-terminal region (aa 479 to 617) and SYVN1 $\Delta$ TM lacking the SyU domain (SYVN1 $\Delta$ TM $\Delta$ SyU) did not interact with PADI4 (Fig. 2B). These results indicated that SYVN1 may interact with PADI4 primarily via the SyU domain.

Inhibition of ubiquitination by PADI4. To assess the association of PADI4 with ubiquitination, the effects of PADI4 overexpression on ubiquitination levels in cells with or without SYVN1 was investigated $(36,39,41)$. HEK293 cells were cotransfected with FLAG-PADI4 and SYVN1 expression vectors, and HA-tagged ubiquitin. In control cells, ubiquitin ladder formations were detected, and SYVN1 expression enhanced this ubiquitination. In contrast, co-expression with PADI4 reduced ubiquitination levels when compared with that observed in control cells (Fig. 3).

Inhibition of the ubiquitination activity of SYVN1 by PADI4. To confirm whether PADI4 inhibited ubiquitination by SYVN1, in vivo ubiquitination assays were performed. HEK293 cells were transfected with His-tagged SYVN1 and FLAG-tagged PADI4 with HA-tagged ubiquitin. SYVN1 was purified with a Ni-sepharose, and auto-ubiquitination was detected. The precipitated SYVN1 was specifically ubiquitinated. PADI4 is a citrullination enzyme, and the cysteine residue at amino acid position 645 in PADI4 is essential for binding with calcium ions and its associated enzymatic activity $(26,47)$. Therefore, the effects of PADI4 enzymatic activity on ubiquitination were assessed using a PADI4 C645S mutant (26). The mutant PADI4 was demonstrated to interact with SYVN1 in the same way as the wild type (Fig. 1). The mutant inhibited ubiquitination

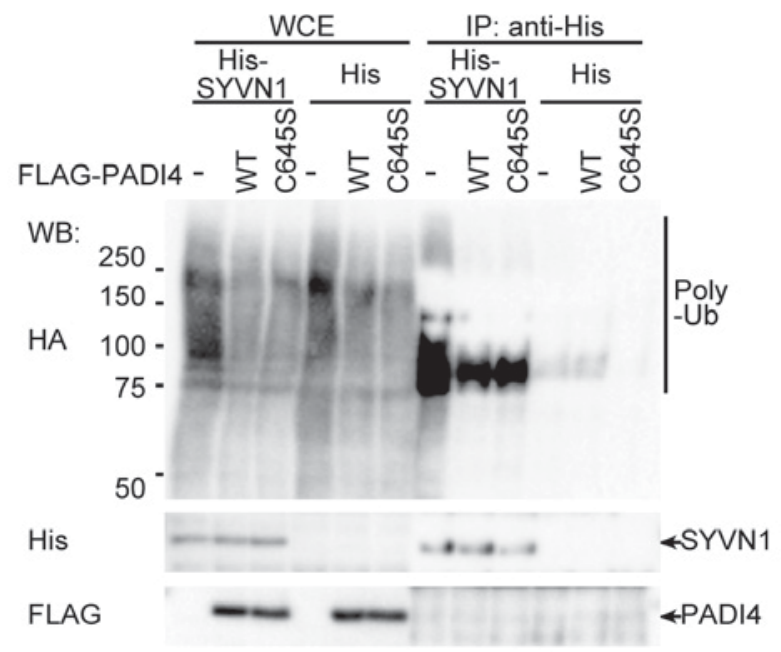

Figure 4. Direct inhibition of SYVN1 ubiquitination by PADI4. HEK293 cells were cotransfected with plasmids containing His-SYVN1 and HA-ubiquitin plus FLAG-PADI4 or its mutant. His-SYVN was precipitated with a Ni-sepharose resin and washed with high-salt buffer. The ubiquitination level of SYVN1 was detected using anti-HA antibodies. SYVN, synoviolin; PADI4, peptidyl-arginine deiminases 4; HA, human influenza hemagglutinin; His, polyhistidine; Ub, ubiquitin; WT, wild type; WCE, whole-cell extracts; WB, western blot analysis.

in HEK293 cells, decreasing ubiquitination to the same level observed for wild type PADI4 (Fig. 4), indicating that the stoichiometric mode rather than the enzymatic activity of PADI4 may be important for inhibition of ubiquitination.

\section{Discussion}

Citrullination affects the structure and function of protein substrates by altering the charge $(25,48)$. Such alterations are thought to induce autoimmune reactions, generating autoantigens in RA. Citrullination is involved in cell growth signals, therefore PADI4 is important for RA pathogenesis (49). The present study revealed that overexpression of PADI4 decreased ubiquitination levels in HEK293 cells and that SYVN1 interacted with PADI4 via the SyU domain and the C-terminal region. The results indicated that PADI4 may suppress the ubiquitination of proteins through associations with E3 ligases. A PADI4 mutant, lacking enzymatic activity, yielded similar results. Thus, PADI4 may prevent ubiquitination via stoichiometric effects, not via enzymatic activity.

A number of post-translational modifications have been reported to interact with each other (6). With regards to PADI and citrullination, it has been demonstrated to interact with acetylation and methylation. Arginine residues are substrates for PADI and protein arginine methyltransferases, therefore citrullination may antagonize methylation modifications and prevent the decondensation of chromatin $(25,50)$. Similarly, ubiquitination and acetylation also compete for lysine residues during the regulation of sodium channels (51). These regulatory mechanisms are based on the competition for enzymatic target residues on the common substrates. However, the association between citrullination and ubiquitination may be mediated via an indirect pathway. Notably, in type 1 diabetes, citrullination and ER stress signals are implicated in the same inflammatory reaction. Binding immunoglobulin protein 
(Bip)/glucose-related protein (GRP)-78, a sensing protein that detects the accumulation of unfolded proteins in the ER, translocates to the plasma membrane where it is modified by PADI2. In this mechanism, the calcium influx induced by inflammatory cytokines and ER stress may be able to activate PADI (52). Citrullinated Bip secreted from cells is recognized as an autoantigen, as previously reported (52-54). Thus, in the present study, inhibition of SYVN1-mediated ubiquitination by PADI4 may represent a novel mechanism for the association between deiminase and ubiquitin ligase.

Notably, PADI4 and SYVN1 regulate the same signaling pathway. The present study demonstrated that SYVN1 binds to p53 in the cytoplasm and negatively regulates p53 signaling via ubiquitination. In addition, downregulation of SYVN1 expression levels has been revealed to decrease the expression of p53 target genes, such as p21 (37). In contrast, upregulation of SYVN1 represses synoviocyte apoptosis in patients with RA, leading to arthritis $(37,38,55)$. Additionally, PADI4 is recruited to target genes with p53 and suppresses transactivation via citrullination of histone H3 (29,56-59). In RA synoviocytes, activation of PADI4 function induces arthritis by inhibiting apoptosis (23). Thus, these reports suggest that activation of PADI4 and SYVN1 signals may enhance cell proliferation by inhibiting apoptosis. However, the results of the present study demonstrated that PADI4 repressed ubiquitination by SYVN1. These results may be inconsistent with the previous reports due to the presence of multiple regulatory mechanisms involving PADI4 and SYVN1.

PADI4 may impair the repression of apoptosis via SYVN1. PADI4 and SYVN1 induce cell proliferation via inhibition of the p53 pathway and apoptosis. In addition, mice deficient in each of these targets have exhibited resistance against the collagen-induced arthritis (36) and glucose-6-phosphate isomerase-induced arthritis models (60), respectively. In addition, synovium derived from patients with RA possess tumor-like characteristics, including their growth ability and morphology (61). However, the proliferation of synoviocytes is not limitless and can be suppressed spontaneously at some stages, unlike the growth of cancer cells (62). One of the mechanisms regulating these features may be Fas-mediated apoptosis in RA synovium (62). Collectively, the results indicated that PADI4 may mediate the balance between the proliferation and death of cells through citrullination and inhibition of ubiquitination by SYVN1. These findings may represent a second mechanism for regulating the overgrowth of the synovium, which is in contrast to tumor cells. Some patients with RA exhibit vasculitis or severe extra-articular manifestations, classified as malignant RA (MRA). The causes of MRA have not yet been clearly defined (63). NETosis, the release of neutrophil extracellular chromatin traps (NETs), has been implicated in these severe rheumatoid disorders $(64,65)$. Autoantigens released outside of the cells during NETosis enhance inflammatory responses as positive feedback, and citrullination induced extracellular PADI4 is accelerated (66). However, the mechanisms mediating the progression of these diseases have not been determined. It is possible that apoptosis of synoviocytes may activate NETosis with inflammatory cytokines derived from the synovium (64). Thus, apoptosis via the crosstalk between PADI4 and SYVN1 may be one of the mechanisms limiting cell proliferation in RA or regulating the exacerbation of RA symptoms.

Another possibility is that inhibition of ubiquitination may mimic the ER stress-repressed state. SYVN1 is implicated in some stages of RA including, inflammation, fibrosis and cartilage destruction through the ERAD system and quality control of substrates $(41,67-69)$. The overexpression of SYVN1 in RA synovium may suppress apoptosis via the activation of the ERAD system and cell proliferation $(41,55)$. Promoting the degradation of unfolded proteins accumulated in the ER by overexpression of SYVN1 results in the reduction of ER stress, therefore ER stress may be reduced or prevented in rheumatoid synovium highly expressing SYVN1. This may lead to inhibition of apoptosis and proliferation of synoviocytes. Namely, PADI4 may cause RA via suppression of the ER stress pathway in addition to the production of autoantigens and demethylation. A number of proteins, including fibrin, fibronectin, vimentin and collagen are citrullinated by PADI in RA (20). The association between PADI family proteins and substrates are unclear. PADI4 is mainly localized in the nucleus and catalyzes histones, however, it has also been detected in sera (24). In addition, in the present study, PADI4 exhibited several functions in addition to its role as a transcriptional repressor. SYVN1 catalyzes the ubiquitination of membrane-anchored proteins and secreted proteins, including cytokines, extracellular matrix proteins and receptors as substrates, and so SYVN1 and PADI4 may catalyze the same substrates. In addition to PADI4, PADI2 has also been implicated in RA. Further studies are required to understand the crosstalk between ubiquitination and citrullination.

In some tumor tissues, PADI4-null mutations are associated with resistance to apoptosis. This phenomenon may be explained by the function of PADI4 as an inhibitor of cell proliferation via citrullination of histone H4R3 under some cellular stresses conditions, such as oxidative stress or radiation (58). PADI4 has been predicted to have the opposite effects in response to cellular stress. However, the roles of PADI4 in ER stress have not been determined. In contrast, SYVN1 has been implicated in oncogenesis and oxidative stress (43). Collectively, these results indicated that cell proliferation may be regulated by PADI4-dependent SYVN1 suppression in response to several types of stress.

The underlying crosstalk mechanisms between PADI4 and SYVN1 remain unclear, and so more extensive analysis is required. PADI4 and SYVN1 serve important roles in a number of diseases, including cancer and autoimmune diseases, such as RA. Understanding of the crosstalk mechanisms associated with PADI4 and SYVN1 may lead to the development of novel therapies for these diseases. In addition, SYVN1 inhibitors have previously been demonstrated to inhibit arthritis and fibrosis in vivo (44-46,70). Thus, these compounds may be useful in the treatment of diseases involving PADI4 or citrullination.

\section{Acknowledgements}

The present study was supported by the Takeda Science Foundation, the Naito Foundation, the Natural Science Scholarship Daiichi-Sankyo Foundation of Life Science, Mitsubishi, the Tanabe Pharma Corporation, Santen 
Pharmaceutical, the Bureau of Social Welfare and Public Health, the Health Labour Sciences Research Grant (grant no. H24-Nanchi-Wakate-010), the Japan Society for the Promotion of Science KAKENHI (grant nos. 22790947, 24791006, 20249052, 23659176, 23659502, 26461476, 26670479, 26461478 and S1411011) and Supporting Positive Activities for Female Researchers.

\section{References}

1. Harris ED Jr: Rheumatoid arthritis. Pathophysiology and implications for therapy. N Engl J Med 322: 1277-1289, 1990.

2. Feldmann M, Brennan FM and Maini RN: Rheumatoid arthritis. Cell 85: 307-310, 1996.

3. Aletaha D, Neogi T, Silman AJ, Funovits J, Felson DT, Bingham CO III, Birnbaum NS, Burmester GR, Bykerk VP, Cohen MD, et al: 2010 Rheumatoid arthritis classification criteria: An American college of rheumatology/European league against rheumatism collaborative initiative. Arthritis Rheum 62 2569-2581, 2010.

4. Gabriel SE: The epidemiology of rheumatoid arthritis. Rheum Dis Clin North Am 27: 269-281, 2001.

5. Smolen JS and Aletaha D: Rheumatoid arthritis therapy reappraisal: Strategies, opportunities and challenges. Nat Rev Rheumatol 11: 276-289, 2015.

6. Yang XJ: Multisite protein modification and intramolecular signaling. Oncogene 24: 1653-1662, 2005.

7. Yamada R, Suzuki A, Chang X and Yamamoto K: Citrullinated proteins in rheumatoid arthritis. Front Biosci 10: 54-64, 2005.

8. Martin-Mola E, Balsa A, Garcia-Vicuna R, Gómez-Reino J, González-Gay MA, Sanmartí R and Loza E: Anti-citrullinated peptide antibodies and their value for predicting responses to biologic agents: A review. Rheumatol Int 36: 1043-1063, 2016.

9. Aggarwal R, Liao K, Nair R, Ringold S and Costenbader KH: Anti-citrullinated peptide antibody assays and their role in the diagnosis of rheumatoid arthritis. Arthritis Rheum 61: 1472-1483, 2009.

10. van Venrooij WJ and Pruijn GJ: Citrullination: A small change for a protein with great consequences for rheumatoid arthritis. Arthritis Res 2: 249-251, 2000.

11. Tarcsa E, Marekov LN, Mei G, Melino G, Lee SC and Steinert PM: Protein unfolding by peptidylarginine deiminase. Substrate specificity and structural relationships of the natural substrates trichohyalin and filaggrin. J Biol Chem 271: 30709-30716, 1996.

12. Vossenaar ER, Zendman AJ, van Venrooij WJ and Pruijn GJ: PAD, a growing family of citrullinating enzymes: Genes, features and involvement in disease. Bioessays 25: 1106-1118, 2003.

13. Klareskog L, Rönnelid J, Lundberg K, Padyukov L and Alfredsson L: Immunity to citrullinated proteins in rheumatoid arthritis. Annu Rev Immunol 26: 651-675, 2008.

14. Schellekens GA, de Jong BA, van den Hoogen FH, van de Putte LB and van Venrooij WJ: Citrulline is an essential constituent of antigenic determinants recognized by rheumatoid arthritis-specific autoantibodies. J Clin Invest 101: 273-281, 1998.

15. Schellekens GA, Visser H, de Jong BA, van den Hoogen FH, Hazes JM, Breedveld FC and van Venrooij WJ: The diagnostic properties of rheumatoid arthritis antibodies recognizing a cyclic citrullinated peptide. Arthritis Rheum 43: 155-163, 2000.

16. Mrabet D, Laadhar L, Haouet S, Sahli H, Zouari B, Makni S and Sellami S: Anomalies of intra-synovial citrullination: Is there any interest in the diagnosis of early rheumatoid arthritis? Rheumatol Int 33: 787-791, 2013.

17. Ikari K, Kuwahara M, Nakamura T, Momohara S, Hara M, Yamanaka H, Tomatsu T and Kamatani N: Association between PADI4 and rheumatoid arthritis: A replication study. Arthritis Rheum 52: 3054-3057, 2005.

18. Foulquier C, Sebbag M, Clavel C, Chapuy-Regaud S, Al Badine R Méchin MC, Vincent C, Nachat R, Yamada M, Takahara H, et al: Peptidyl arginine deiminase type 2(PAD-2) and PAD-4 but not PAD-1, PAD-3, and PAD-6 are expressed in rheumatoid arthritis synovium in close association with tissue inflammation. Arthritis Rheum 56: 3541-3553, 2007.

19. Chang X, Xia Y, Pan J, Meng Q, Zhao Y and Yan X: PADI2 is significantly associated with rheumatoid arthritis. PLoS One 8 : e81259, 2013.
20. Anzilotti C, Pratesi F, Tommasi C and Migliorini P: Peptidylarginine deiminase 4 and citrullination in health and disease. Autoimmun Rev 9: 158-160, 2010.

21. Jones JE, Causey CP, Knuckley B, Slack-Noyes JL and Thompson PR: Protein arginine deiminase 4 (PAD4): Current understanding and future therapeutic potential. Curr Opin Drug Discov Devel 12: 616-627, 2009.

22. Suzuki A, Yamada R, Chang X, Tokuhiro S, Sawada T, Suzuki M, Nagasaki M, Nakayama-Hamada M, Kawaida R, Ono M, et al: Functional haplotypes of PADI4, encoding citrullinating enzyme peptidylarginine deiminase 4 , are associated with rheumatoid arthritis. Nat Genet 34: 395-402, 2003.

23. Chang X, Yamada R, Suzuki A, Sawada T, Yoshino S, Tokuhiro S and Yamamoto K: Localization of peptidylarginine deiminase 4 (PADI4) and citrullinated protein in synovial tissue of rheumatoid arthritis. Rheumatology (Oxford) 44: 40-50, 2005.

24. Mastronardi FG, Wood DD, Mei J, Raijmakers R, Tseveleki V, Dosch HM, Probert L, Casaccia-Bonnefil P and Moscarello MA: Increased citrullination of histone $\mathrm{H} 3$ in multiple sclerosis brain and animal models of demyelination: A role for tumor necrosis factor-induced peptidylarginine deiminase 4 translocation. J Neurosci 26: 11387-11396, 2006.

25. Cuthbert GL, Daujat S, Snowden AW, Erdjument-Bromage H, Hagiwara T, Yamada M, Schneider R, Gregory PD, Tempst P, Bannister AJ and Kouzarides T: Histone deimination antagonizes arginine methylation. Cell 118: 545-553, 2004.

26. Wang Y, Wysocka J, Sayegh J, Lee YH, Perlin JR, Leonelli L, Sonbuchner LS, McDonald CH, Cook RG, Dou Y, et al: Human PAD4 regulates histone arginine methylation levels via demethylimination. Science 306: 279-283, 2004.

27. Wang Y, Li M, Stadler S, Correll S, Li P, Wang D, Hayama R, Leonelli L, Han H, Grigoryev SA, et al: Histone hypercitrullination mediates chromatin decondensation and neutrophil extracellular trap formation. J Cell Biol 184: 205-213, 2009.

28. Li P, Li M, Lindberg MR, Kennett MJ, Xiong N and Wang Y: PAD4 is essential for antibacterial innate immunity mediated by neutrophil extracellular traps. J Exp Med 207: 1853-1862, 2010.

29. Tanikawa C, Ueda K, Nakagawa H, Yoshida N, Nakamura Y and Matsuda K: Regulation of protein Citrullination through p53/PADI4 network in DNA damage response. Cancer Res 69: 8761-8769, 2009

30. Yoo SA, You S, Yoon HJ, Kim DH, Kim HS, Lee K, Ahn JH, Hwang D, Lee AS, Kim KJ, et al: A novel pathogenic role of the ER chaperone GRP78/BiP in rheumatoid arthritis. J Exp Med 209: 871-886, 2012.

31. Manié SN, Lebeau J and Chevet E: Cellular mechanisms of endoplasmic reticulum stress signaling in health and disease. 3. Orchestrating the unfolded protein response in oncogenesis: An update. Am J Physiol Cell Physiol 307: C901-C907, 2014.

32. Hotamisligil GS and Erbay E: Nutrient sensing and inflammation in metabolic diseases. Nat Rev Immunol 8: 923-934, 2008.

33. Park YJ, Yoo SA and Kim WU: Role of endoplasmic reticulum stress in rheumatoid arthritis pathogenesis. J Korean Med Sci 29: 2-11, 2014.

34. Gao B, Lee SM, Chen A, Zhang J, Zhang DD, Kannan K, Ortmann RA and Fang D: Synoviolin promotes IRE1 ubiquitination and degradation in synovial fibroblasts from mice with collagen-induced arthritis. EMBO Rep 9: 480-485, 2008.

35. Connor AM, Mahomed N, Gandhi R, Keystone EC and Berger SA: TNF $\alpha$ modulates protein degradation pathways in rheumatoid arthritis synovial fibroblasts. Arthritis Res Ther 14: R62, 2012.

36. Amano T, Yamasaki S, Yagishita N, Tsuchimochi K, Shin H, Kawahara K, Aratani S, Fujita H, Zhang L, Ikeda R, et al: Synoviolin/Hrd1, an E3 ubiquitin ligase, as a novel pathogenic factor for arthropathy. Genes Dev 17: 2436-2449, 2003.

37. Yamasaki S, Yagishita N, Sasaki T, Nakazawa M, Kato Y, Yamadera T, Bae E, Toriyama S, Ikeda R, Zhang L, et al: Cytoplasmic destruction of p53 by the endoplasmic reticulum-resident ubiquitin ligase 'Synoviolin'. Embo J 26: 113-122, 2007.

38. Yamasaki S, Yagishita N, Nishioka K and Nakajima T: The roles of synoviolin in crosstalk between endoplasmic reticulum stress-induced apoptosis and p53 pathway. Cell Cycle 6: 1319-1323, 2007.

39. Toh ML, Marotte H, Blond JL, Jhumka U, Eljaafari A, Mougin B and Miossec P: Overexpression of synoviolin in peripheral blood and synoviocytes from rheumatoid arthritis patients and continued elevation in nonresponders to infliximab treatment. Arthritis Rheum 54: 2109-2118, 2006. 
40. Klaasen R, Wijbrandts CA, van Kuijk AW, Pots D, Gerlag DM and Tak PP: Synovial synoviolin in relation to response to TNF blockade in patients with rheumatoid arthritis and psoriatic arthritis. Ann Rheum Dis 71: 1260-1261, 2012.

41. Yagishita N, Yamasaki S, Nishioka K and Nakajima T: Synoviolin, protein folding and the maintenance of joint homeostasis. Nat Clin Pract Rheumatol 4: 91-97, 2008.

42. Hasegawa D, Fujii R, Yagishita N, Matsumoto N, Aratani S, Izumi T, Azakami K, Nakazawa M, Fujita H, Sato T, et al: E3 ubiquitin ligase synoviolin is involved in liver fibrogenesis. PLoS One 5: e13590, 2010.

43. Wu T, Zhao F, Gao B, Tan C, Yagishita N, Nakajima T, Wong PK, Chapman E, Fang D and Zhang DD: Hrd1 suppresses Nrf2-mediated cellular protection during liver cirrhosis. Genes Dev 28: 708-722, 2014.

44. Nakajima F, Aratani S, Fujita H, Yagishita N, Ichinose S, Makita K, Setoguchi Y and Nakajima T: Synoviolin inhibitor LS-102 reduces endoplasmic reticulum stress-induced collagen secretion in an in vitro model of stress-related interstitial pneumonia. Int J Mol Med 35: 110-116, 2015.

45. Fujita H, Yagishita N, Aratani S, Saito-Fujita T, Morota S, Yamano Y, Hansson MJ, Inazu M, Kokuba H, Sudo K' et al: The E3 ligase synoviolin controls body weight and mitochondrial biogenesis through negative regulation of PGC-1 $\beta$. EMBO J 34 1042-1055, 2015.

46. Yagishita N, Aratani S, Leach C, Amano T, Yamano Y Nakatani K, Nishioka K and Nakajima T: RING-finger type E3 ubiquitin ligase inhibitors as novel candidates for the treatment of rheumatoid arthritis. Int J Mol Med 30: 1281-1286, 2012

47. Arita K, Hashimoto H, Shimizu T, Nakashima K, Yamada M and Sato M: Structural basis for $\mathrm{Ca}(2+)$-induced activation of human PAD4. Nat Struct Mol Biol 11: 777-783, 2004.

48. Knuckley B, Bhatia M and Thompson PR: Protein arginine deiminase 4: Evidence for a reverse protonation mechanism Biochemistry 46: 6578-6587, 2007.

49. Vossenaar ER, Nijenhuis S, Helsen MM, van der Heijden A, Senshu T, van den Berg WB, van Venrooij WJ and Joosten LA: Citrullination of synovial proteins in murine models of rheumatoid arthritis. Arthritis Rheum 48: 2489-2500, 2003.

50. Raijmakers R, Zendman AJ, Egberts WV, Vossenaar ER, Raats J, Soede-Huijbregts C, Rutjes FP, van Veelen PA, Drijfhout JW and Pruijn GJ: Methylation of arginine residues interferes with citrullination by peptidylarginine deiminases in vitro. J Mol Biol 367: $1118-1129,2007$

51. Butler PL, Staruschenko A and Snyder PM: Acetylation stimulates the epithelial sodium channel by reducing its ubiquitination and degradation. J Biol Chem 290: 12497-13503, 2015.

52. Rondas D, Crèvecoeur I, D'Hertog W, Ferreira GB, Staes A, Garg AD, Eizirik DL, Agostinis P, Gevaert K, Overbergh L and Mathieu C: Citrullinated glucose-regulated protein 78 is an autoantigen in type 1 diabetes. Diabetes 64: 573-586, 2015.

53. Skriner K, Adolph K, Jungblut PR and Burmester GR: Association of citrullinated proteins with synovial exosomes. Arthritis Rheum 54: 3809-3814, 2006

54. Goëb V, Thomas-L'Otellier M, Daveau R, Charlionet R, Fardellone P, Le Loët X, Tron F, Gilbert D and Vittecoq O: Candidate autoantigens identified by mass spectrometry in early rheumatoid arthritis are chaperones and citrullinated glycolytic enzymes. Arthritis Res Ther 11: R38, 2009

55. Yamasaki S, Yagishita N, Tsuchimochi K, Kato Y, Sasaki T, Amano T, Beppu M, Aoki H, Nakamura H, Nishioka K and Nakajima T: Resistance to endoplasmic reticulum stress is an acquired cellular characteristic of rheumatoid synovial cells. Int J Mol Med 18: 113-117, 2006.
56. Yao H, Li P, Venters BJ, Zheng S, Thompson PR, Pugh BF and Wang Y: Histone Arg modifications and p53 regulate the expression of OKL38, a mediator of apoptosis. J Biol Chem 283: 20060-20068, 2008

57. Li P, Yao H, Zhang Z, Li M, Luo Y, Thompson PR, Gilmour DS and Wang Y: Regulation of p53 target gene expression by peptidylarginine deiminase 4. Mol Cell Biol 28: 4745-4758, 2008.

58. Tanikawa C, Espinosa M, Suzuki A, Masuda K, Yamamoto K, Tsuchiya E, Ueda K, Daigo Y, Nakamura Y and Matsuda K: Regulation of histone modification and chromatin structure by the p53-PADI4 pathway. Nat Commun 3: 676, 2012.

59. Liu GY, Liao YF, Chang WH, Liu CC, Hsieh MC, Hsu PC, Tsay GJ and Hung HC: Overexpression of peptidylarginine deiminase IV features in apoptosis of haematopoietic cells Apoptosis 11: 183-196, 2006.

60. Seri Y, Shoda H, Suzuki A, Matsumoto I, Sumida T, Fujio K and Yamamoto K: Peptidylarginine deiminase type 4 deficiency reduced arthritis severity in a glucose-6-phosphate isomerase-induced arthritis model. Sci Rep 5: 13041, 2015.

61. Moran M, Fang C and Paul A: Rheumatoid arthritis presenting as an invasive soft-tissue tumour. Arch Orthop Trauma Surg 122: 538-540, 2002

62. Nakajima T, Aono H, Hasunuma T, Yamamoto K, Shirai T, Hirohata K and Nishioka K: Apoptosis and functional Fas antigen in rheumatoid arthritis synoviocytes. Arthritis Rheum 38: 485-491, 1995.

63. Nobunaga M: Malignant rheumatoid arthritis. Nihon Rinsho 50: 597-602, 1992 (In Japanese).

64. Khandpur R, Carmona-Rivera C, Vivekanandan-Giri A, Gizinski A, Yalavarthi S, Knight JS, Friday S, Li S, Patel RM, Subramanian V, et al: NETs are a source of citrullinated autoantigens and stimulate inflammatory responses in rheumatoid arthritis. Sci Transl Med 5: 178ra40, 2013.

65. Dwivedi N, Upadhyay J, Neeli I, Khan S, Pattanaik D, Myers L, Kirou KA, Hellmich B, Knuckley B and Thompson PR, et al: Felty's syndrome autoantibodies bind to deiminated histones and neutrophil extracellular chromatin traps. Arthritis Rheum 64: 982-992, 2012

66. Spengler J, Lugonja B, Jimmy Ytterberg A, Zubarev RA, Creese AJ, Pearson MJ, Grant MM, Milward M, Lundberg K, Buckley CD, et al: Release of active peptidyl arginine deiminases by neutrophils can explain production of extracellular citrullinated autoantigens in rheumatoid arthritis synovial fluid. Arthritis Rheumatol 67: 3135-3145, 2015.

67. Li L, Shen Y, Ding Y, Liu Y, Su D and Liang X: Hrd1 participates in the regulation of collagen I synthesis in renal fibrosis. Mol Cell Biochem 386: 35-44, 2014.

68. Toh ML, Gonzales G, Koenders MI, Tournadre A, Boyle D, Lubberts E, Zhou Y, Firestein GS, van den Berg WB and Miossec P: Role of interleukin 17 in arthritis chronicity through survival of synoviocytes via regulation of synoviolin expression. PLoS One 5: e13416, 2010.

69. Burr ML, van den Boomen DJ, Bye H, Antrobus R, Wiertz EJ and Lehner PJ: MHC class I molecules are preferentially ubiquitinated on endoplasmic reticulum luminal residues during HRD1 ubiquitin E3 ligase-mediated dislocation. Proc Natl Acad Sci USA 110: 14290-14295, 2013.

70. Bianchini E, Fanin M, Mamchaoui K, Betto R and Sandonà D: Unveiling the degradative route of the V247M $\alpha$-sarcoglycan mutant responsible for LGMD-2D. Hum Mol Genet 23 3746-3758, 2014 